\title{
Respuesta terapéutica de Plasmodium vivax a la cloroquina, en Riberalta, Guayaramerín y Yacuiba, Bolivia
}

\author{
Arletta Añez ${ }^{1}$, Dennis Navarro-Costa ${ }^{1}$, Omar Yucra², Cecilia Garnica ${ }^{3}$, Viviana Melgar ${ }^{3}$, \\ Manuel Moscoso ${ }^{3}$, Ricardo Arteaga ${ }^{4}$, Gladys Nakao ${ }^{5}$ \\ 1 Organización Panamericana de la Salud, Organización Mundial de la Salud, La Paz, Bolivia \\ 2 Centro de Salud El Palmar, Yacuiba-Tarija, Bolivia \\ ${ }^{3}$ Instituto Nacional de Laboratorios en Salud, Centro Nacional de Análisis de Medicamentos y Tóxicos \\ CONCAMYT, La Paz, Bolivia \\ ${ }^{4}$ Hospital San Gabriel, Guayaramerín-Beni, Bolivia \\ ${ }^{5}$ Laboratorio Programa de Malaria, Riberalta-Beni, Bolivia
}

Introducción. La determinación de la eficacia de la cloroquina contra Plasmodium vivax permite mejorar la capacidad de vigilancia de la resistencia a los antipalúdicos.

Objetivo. Evaluar la eficacia terapéutica de la cloroquina como tratamiento de malaria no complicada por P. vivax en Riberalta, Guayaramerín y Yacuiba, Bolivia.

Materiales y métodos. Se llevó a cabo un estudio de la eficacia in vivo en pacientes mayores de cinco años; se suministró cloroquina (25 mg/kg en tres días) y se hizo seguimiento por 28 días, midiendo los niveles de cloroquina en sangre y desetilcloroquina, el día dos y el día de registro de reaparición de parasitemia. Para la evaluación de la incidencia acumulada de falla del tratamiento, se usó el análisis de supervivencia de Kaplan-Meier.

Resultados. Se estudiaron 223 pacientes (Riberalta, 84; Guayaramerín, 80; Yacuiba, 59). Las medias de densidad parasitaria (formas asexuadas) del día 0 en Riberalta fueron de 6.147, en Guayaramerín, 4.251, y en Yacuiba, 5.214 parásitos/ $\mu$ l de sangre. En el mismo orden, los promedios de concentraciones sanguíneas de cloroquina-desetilcloroquina del día 2 fueron de 783, 817 y $815 \mathrm{ng} / \mathrm{ml}$. Mientras en Yacuiba no se presentaron fracasos terapéuticos, en Riberalta ocurrieron con frecuencia de 6,2 \% y en Guayaramerín de 10 \%. Los valores de cloroquina y desetilcloroquina en sangre de pacientes con fracaso terapéutico fueron menores de $70 \mathrm{ng} / \mathrm{ml}$ en el día de reaparición de parasitemia.

Conclusión. No se evidenció resistencia de $P$. vivax a la cloroquina en las tres regiones de evaluación en Bolivia. Se requieren mayores estudios de la concentración de la cloroquina en sangre.

Palabras clave: Plasmodium vivax, malaria, cloroquina, antipalúdicos, Bolivia.

doi: http://dx.doi.org/10.7705/biomedica.v32i4.750

\section{Therapeutic response of Plasmodium vivax to chloroquine in Bolivia}

Introduction. Knowledge of the therapeutic efficacy of chloroquine for Plasmodium vivax infections improves the capacity for surveillance of anti-malarial drug resistance.

Objective. The therapeutic efficacy of chloroquine as treatment was evaluated for uncomplicated Plasmodium vivax malaria in Bolivia.

Materials and methods. An in vivo efficacy study of chloroquine was undertaken in three regions of Bolivia--Riberalta, Guayaramerín and Yacuiba. Two hundred and twenty-three patients (84, 80, and 59 in the three regions, respectively) aged over 5 years old were administered with chloroquine (25 $\mathrm{mg} / \mathrm{kg} /$ three days) and followed for 28 days. Blood levels of chloroquine and desethylchloroquine were measured on day 2 and on the day of reappearance of parasitemia. The cumulative incidence of treatment failure was calculated using the Kaplan and Meier survival analysis.

Results. The mean parasitemias (asexual) on day 0 were 6,147 parasites/ $\mu$ l of blood in the Riberalta population, 4,251 in Guayaramerín and 5,214 in Yacuiba. The average blood concentrations of

\section{Contribución de los autores:}

Arletta Añez: diseñó el estudio, coordinó las actividades y el cumplimiento de los pacientes en Riberalta.

Dennis Navarro-Costa: capacitó los equipos de investigación y supervisó el estudio.

Arletta Añez y Dennis Navarro-Costa: analizaron los resultados y redactaron el manuscrito.

Omar Yucra y Ricardo Arteaga: cumplimiento de pacientes y coordinación de actividades en la localidad de Yacuiba.

Cecilia Garnica: medición de la cloroquina en sangre e interpretación de los resultados.

Viviana Melgar y Manuel Moscoso: extracción de la muestra para la medición de la cloroquina en sangre; redactaron y analizaron los resultados.

Gladys Nakao: responsable del diagnóstico microscópico y del control de calidad en Riberalta. 
chloroquine-desethylchloroquine during day 2 were 783, 817, and $815 \mathrm{ng} / \mathrm{ml}$, respectively. No treatment failures were observed in Yacuiba, whereas in Riberalta and Guayaramerín, the frequencies of treatment failures were $6.2 \%$ and $10 \%$. Blood levels of chloroquine and desethylchloroquine in patients with treatment failure showed values below $70 \mathrm{ng} / \mathrm{ml}$ on the day of reappearance of parasitemia.

Conclusion. Resistance of Plasmodium vivax to chloroquine was not demonstrated in three regions of Bolivia.

Key words: Plasmodium vivax, malaria, chloroquine, antimalarials, Bolivia. doi: http://dx.doi.org/10.7705/biomedica.v32i4.750

En el mundo, la malaria aún representa un serio riesgo para la salud pública (1). En la región de las Américas, a pesar de que según los datos de la Organización Panamericana de la Salud (OPS), en el 2008 disminuyó el registro de casos en 30,7 $\%$ en relación con el 2000 , la malaria o paludismo continúa siendo una entidad que está presente en una amplia región del continente y cuyo riesgo de transmisión se halla ligado al comportamiento de las poblaciones, el cual está influenciado en forma importante por factores sociales, culturales, económicos y políticos $(2,3)$. La OPS también indica que la infección por Plasmodium vivax es la predominante y que representa el $75 \%$ de los casos de malaria reportados (3).

La quimioterapia antipalúdica se constituye en una de las principales herramientas para la lucha contra esta enfermedad y, a pesar de los esfuerzos para frenar la aparición, diseminación e incremento de la resistencia a los antipalúdicos, la resistencia a la cloroquina representa una preocupación particular (4).

Los primeros casos de $P$. vivax resistentes a la cloroquina fueron reportados en dos soldados australianos en Papua, Nueva Guinea, en 1989 (5). También, se reportaron casos en Indonesia y en el este de Asia, donde se registraron fallas terapéuticas en $15 \%$ a $44 \%$ de los casos de $P$. vivax tratados con cloroquina (6-8) y, de igual forma, en el noreste de África se encuentran casos de resistencia de $P$. vivax a la cloroquina $(9,10)$.

En Sudamérica, los primeros casos de resistencia o falla terapéutica de este medicamento en infecciones por $P$. vivax se registraron en Guyana, donde en tres pacientes con niveles séricos adecuados de cloroquina persistió la parasitemia (11). Desde

Correspondencia:

Arletta Añez, OPS/OMS, Plaza España, Edificio Barcelona, piso 6, La Paz, Bolivia.

Teléfonos: (591) 79699943 y (5912) 2412465

aanez@bol.ops-oms.org

Recibido: 13/05/11; aceptado:02/06/12 entonces y hasta la fecha, se registraron casos de falla terapéutica en Brasil y Colombia $(12,13)$ y, de resistencia confirmada, mediante la evaluación de los niveles séricos de cloroquina en sangre, en el Perú $(2 \%)(14,15)$ y en el Brasil $(10,1 \%)(16)$. En Bolivia, en el 2003 se hizo un estudio in vivo de la eficacia de la cloroquina, en la localidad de Palmar Chico en Yacuiba, y se demostró $100 \%$ de eficacia del antipalúdico para infecciones por $P$. vivax, hallazgo igual al obtenido en la Costa Pacífica de Colombia $(17,18)$.

Actualmente, el esquema de tratamiento de primera línea recomendado para infecciones no complicadas por $P$. vivax por el Programa Nacional de Control de la Malaria de Bolivia, es de 25 mg/ $\mathrm{kg}$ de cloroquina por vía oral, suministrado en tres días, más $0,5 \mathrm{mg} / \mathrm{kg}$ de primaquina por vía oral, durante siete días (19).

Teniendo en cuenta la importancia del paludismo en el perfil epidemiológico de Bolivia, además de las características del proceso salud-enfermedad que dan lugar a la presencia de la enfermedad, para el Programa Nacional de Control de la Malaria es importante mantener activala vigilancia de la eficacia de los fármacos antipalúdicos, recomendada por la OMS (20); es con este propósito que se hace esta evaluación in vivo, para conocer el grado actual de la eficacia de la cloroquina para el tratamiento de la malaria por $P$. vivax en Bolivia.

\section{Materiales y métodos}

\section{Lugar del estudio}

El estudio fue desarrollado, de manera independiente, en los centros de referencia para malaria localizados en Riberalta y Guayaramerín, municipios pertenecientes al departamento del Beni, en el noreste amazónico del país, y en el Centro de Salud de El Palmar, localidad perteneciente al municipio de Yacuiba del departamento de Tarija, ubicado al sur de Bolivia, limítrofe con la República de Argentina (21) (figura 1). Con relación a la presencia dle paludismo en dichas localidades, se puede decir que en la región amazónica, donde la transmisión 


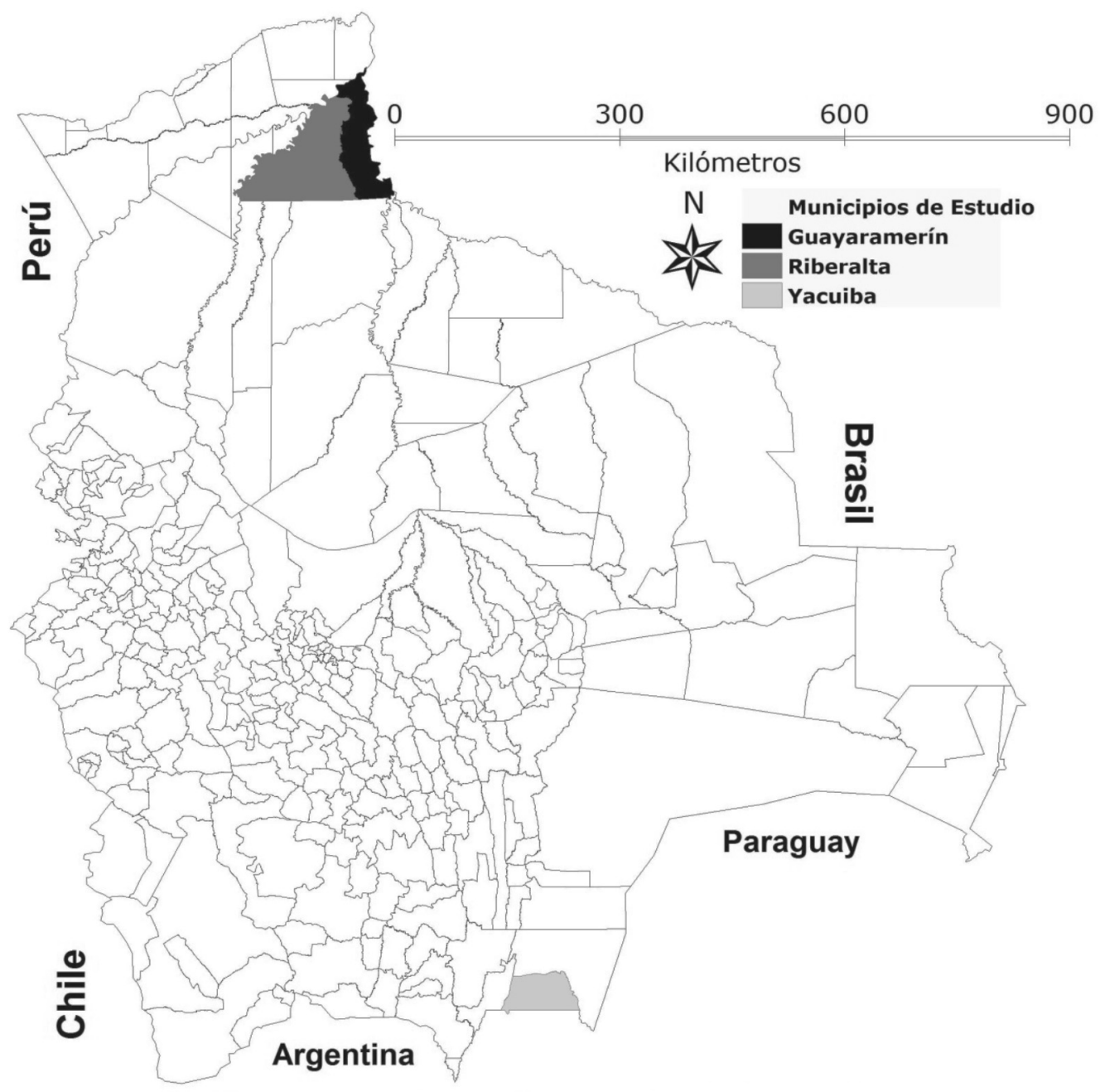

Figura 1. Mapa de Bolivia y municipios del estudio

ocurre durante todo el año y donde se localizan Riberalta y Guayaramerín, se registran infecciones por $P$. falciparum y vivax; mientras que en el sur de Bolivia, donde está Yacuiba, solamente se presentan casos de $P$. vivax, estando la transmisión estacional supeditada al periodo lluvioso del año, que se da de octubre a abril (22).

\section{Diseño}

Se siguió la metodología estandarizada de la OPS/OMS, la guía práctica para estudios in vivo de eficacia de los medicamentos antipalúdicos en las Américas y otras recomendaciones para la evaluación in vivo de la resistencia a los fármacos antipalúdicos (23-25).

El tamaño muestral se estableció según las directrices de OMS/OPS, determinado por la proporción de fracasos terapéuticos de Sudamérica, correspondiente a no más de $5 \%$, con un nivel de confianza del $95 \%$ y una precisión del $5 \%$, por lo cual se decidió incluir en el estudio a 73 pacientes en las tres localidades de estudio.

Se incluyeron en el estudio pacientes mayores de cinco años con diagnóstico de monoparasitemia por $P$. vivax, con una densidad parasitaria de formas asexuadas entre 250 y 100.000 parásitos/ $\mu l$ de sangre y con temperatura mayor de $37,5^{\circ} \mathrm{C}$ o, en su defecto, con antecedentes de alza térmica en las últimas 48 horas.

El diagnóstico microscópico se hizo según las técnicas del Programa Nacional de Control de la Malaria (26) (gota gruesa, extendido sanguíneo con tinción de Giemsa) y la densidad parasitaria se 
calculó por microlitro de sangre, mediante la división del número de parásitos contados, multiplicado por 6.000 y dividido entre 200 leucocitos contados.

Cada muestra fue leída por dos microscopistas entrenados y, en caso de una discrepancia mayor de $50 \%$ del resultado de la densidad parasitaria, la muestra fue leída por un tercer microscopista y el resultado final se obtuvo del promedio de los dos microscopistas concordantes, para posteriormente registrar las densidades parasitarias de formas asexuadas jóvenes del párasito.

Se excluyeron del estudio las mujeres con embarazo confirmado mediante pruebas rápidas de embarazo (detección de gonadotropina coriónica humana en orina), los pacientes con signos y síntomas de malaria grave, con historia de alergia a medicamentos antipalúdicos o con presencia concomitante de enfermedades graves o crónicas, y los pacientes que residieran a más de 30 minutos de distancia del centro de reclutamiento. Los que aceptaron participar en el estudio, después de la firma del consentimiento informado, fueron seguidos durante 28 días por parte de los equipos de investigación. El día denle que se hizo el diagnóstico, se denominó día 0 (cero).

Para el tratamiento se utilizó fosfato de cloroquina (Lote 1365, CIPA S.A. Lima, Perú) a dosis de 25 $\mathrm{mg} / \mathrm{kg}$ administrada por vía oral en tres días, así: los días 0 y 1 del estudio, se administraron $10 \mathrm{mg} /$ $\mathrm{kg}$, y el día 2 del estudio, $5 \mathrm{mg} / \mathrm{kg}$, procedimiento que fue estrictamente supervisado por el personal del estudio. A los que presentaron vómito después de media hora de suministrado el medicamento, se les administró un nuevo ciclo de tratamiento con la misma dosis, y aquellos con más de dos episodios de vómito se excluyeron del estudio.

A todos los pacientes se les pidió retornar los días $1,2,3,7,14,21$ y 28 de seguimiento, para dar el tratamiento supervisado en los días correspondientes y hacer valoración clínica, control de temperatura y medición de la densidad parasitaria, según lo establecido en el protocolo de investigación.

Después de los 28 días de seguimiento, todos fueron tratados con $0,5 \mathrm{mg} / \mathrm{kg}$ de primaquina al día por vía oral, durante siete días, de acuerdo con el esquema de tratamiento del programa de malaria del país.

\section{Cromatografía líquida de alta resolución}

La segunda fase del estudio consiste en la medición de la concentración de la cloroquina en sangre total, mediante cromatografía líquida de alta resolución (High-Performance Liquid Chromatography, HPLC). Para tal efecto, el día 2 del seguimiento, antes de la administración de la última dosis de tratamiento, se recolectaron $2 \mathrm{ml}$ de sangre mediante venopunción. Se hizo el mismo procedimiento en el día de control en el que se encontró reaparición de parasitemia, considerándose tal día como el día de falla terapéutica. Las muestras obtenidas se refrigeraron a $4{ }^{\circ} \mathrm{C}$ hasta el día de la medición de los niveles sanguíneos de cloroquina y de su metabolito principal, la desetilcloroquina, medidos por HPLC (27). Una concentración entre 70 y 90 $\mathrm{ng} / \mathrm{ml}$ de cloroquina, más su metabolito principal, desetilcloroquina, es capaz de eliminar el total de formas asexuadas y sexuadas de $P$. vivax de la sangre $(28,29)$. Una concentración de cloroquina y desetil-cloroquina mayor de $100 \mu \mathrm{g} / \mathrm{ml}$, en presencia de parasitemia después de la conclusión del tratamiento con 1,5 g de cloroquina, confirma la resistencia del parásito a dicho fármaco (30-32).

Las condiciones de la cromatografía con que se trabajó fueron: columna cromatográfica, Agilent Zorbax SIL, empaque silano (correspondiente a L3 según USP); detector de fluorescencia, emisión $380 \mathrm{~nm}$, excitación $320 \mathrm{~nm}$; fase móvil A, metanol y dietilamina (100:0,3); fase móvil B, n-hexano, metilterbutileter y dietilamina (1:1:0,003); gradiente de flujo, 1,0 m por minuto; volumen de inyección, 7 $\mu \mathrm{l}$, y temperatura de columna, $30^{\circ} \mathrm{C}$.

\section{Análisis estadístico}

Los datos obtenidos se procesaron mediante una herramienta diseñada en Microsoft Exce ${ }^{\circledR}(33)$, para el análisis de la supervivencia de Kaplan-Meier. Para el análisis de frecuencia y de comparación de medias, se utilizó el programa estadístico SPSS ${ }^{\circledR}$, versión 14 (SPSS Inc., Chicago, IL).

\section{Elaboración de mapas}

Para la elaboración del mapa de referencia, se usó el software libre Terraview 3.6.0.

\section{Aspectos éticos}

Para la elaboración del presente estudio, se siguieron las recomendaciones del Comité Nacional de Bioética de Bolivia para estudios de investigación biomédica; la participación de los pacientes fue voluntaria, avalada por la firma del consentimiento informado. En caso de los menores de edad, se obtuvo su asentimiento informado, además del consentimiento informado de los padres. El protocolo de estudio fue aprobado por el comité anteriormente citado. 


\section{Resultados}

\section{Yacuiba}

El estudio en el municipio de Yacuiba se desarrolló entre septiembre de 2006 y junio de 2007. Se incluyeron 59 pacientes en el estudio. La media de edad encontrada fue de 19 años,y la media geométrica de la densidad parasitaria del día 0 fue de 5.158 parásitos/ $\mu$ y de 719 parásitos sexuados/ $\mu \mathrm{l}$ (cuadro 1).

En el día 3 de seguimiento, 24 horas después de la administración de la última dosis de cloroquina, se observó la eliminación del $100 \%$ de las formas asexuadas. En los controles de los días 7, 14, 21 y 28 de seguimiento, no se observó ninguna forma asexuada del parásito. Dos pacientes abandonaron el estudio, uno en el día 21 y otro en el día 28, mientras que los 57 pacientes restantes completaron el seguimiento de 28 días.

La concentración media de cloroquina y desetilcloroquina en el día 2, antes de la última dosis de cloroquina, fue de $815 \mathrm{ng} / \mathrm{ml}$, registrando un valor mínimo de 144,49 ng/ml y uno máximo de 1.924 $\mathrm{ng} / \mathrm{ml}$.

Basado en el análisis de supervivencia de KaplanMeier, la incidencia acumulada de falla terapéutica fue de 0 , durante todo el seguimiento.

No fue necesaria la toma de una segunda muestra de sangre para la medición de cloroquina y desetilcloroquina porque, después del día 3 de seguimiento, ninguno de los 57 pacientes presentó parasitemia nuevamente.

\section{Riberalta}

En el municipio de Riberalta, entre junio de 2006 y abril de 2007, se incluyeron 84 pacientes en el estudio.
La media de edad encontrada fue de 27 años y las medias geométricas de las densidades parasitarias del día 0 fueron de 6.147 parásitos asexuado/ $\mu$ y 520 parásitos sexuados / $\mu$ l (cuadro 1).

Luego de finalizar la administración de cloroquina, en el día 3 del seguimiento se observó la eliminación del $100 \%$ de las formas asexuadas. En el día 21 de seguimiento, un paciente presentó formas asexuadas del parásito; lo mismo aconteció con otros cuatro pacientes en el día 28 de seguimiento. Dos pacientes abandonaron el estudio en el día 3 y otro fue excluido el día 14 por presentar paludismo mixto.

La concentración media en sangre de cloroquina y desetilcloroquina, el día 2 antes de la última dosis de cloroquina, fue de $783 \mathrm{ng} / \mathrm{ml}$ (valor mínimo de 224,73 y máximo de $1.991,19$ ).

Con base en el análisis de supervivencia de KaplanMeier, la incidencia acumulada de falla terapéutica fue de 0,062 (6,2\%) (IC $\left.{ }_{95 \%}: 0,026-0,142\right)$.

La concentración de cloroquina y desetilcloroquina en sangre de los cinco pacientes de Riberalta que presentaron falla terapéutica durante los días del seguimiento, mostró valores menores de $70 \mathrm{ng} / \mathrm{ml}$.

\section{Guayaramerín}

En el municipio de Guayaramerín, de todos los pacientes atendidos entre agosto de 2006 y febrero de 2007, se incluyeron 80 en el estudio. La media de edad encontrada fue de 24 años y la media geométrica de la densidad parasitaria del día 0 fue de 4.251 parásitos/ $\mu$ l (cuadro 1).

Después de finalizar la administración de cloroquina, en el día 3 del seguimiento se observó la eliminación del $100 \%$ de las formas asexuadas.

Cuadro 1. Datos de inclusión de pacientes al estudio y concentración de cloroquina y desetilcloroquina, segundo día de seguimiento

\begin{tabular}{|c|c|c|c|c|c|c|}
\hline $\begin{array}{l}\text { Lugar de } \\
\text { estudio }\end{array}$ & Sexo & $\begin{array}{l}\text { Número de } \\
\text { pacientes }\end{array}$ & $\begin{array}{l}\text { Edad promedio } \\
\text { en años (rango) }\end{array}$ & $\begin{array}{l}\text { Temperatura } \\
\text { promedio } \\
\left({ }^{\circ} \mathrm{C}\right) \mathrm{DO}^{\star}\end{array}$ & $\begin{array}{l}\text { Parasitemia } \\
\text { promedio } \\
\text { promedio D0 } \\
\text { (parásitos/ul) }\end{array}$ & 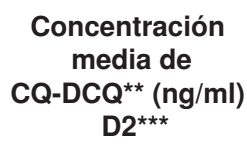 \\
\hline \multirow[t]{2}{*}{ Guayaramerín } & $\mathrm{F}$ & 34 & 22 (8-37) & 37 & 4.500 & 890 \\
\hline & M & 46 & $27(8-60)$ & 38 & 3.755 & 760 \\
\hline \multirow[t]{2}{*}{ Riberalta } & $\mathrm{F}$ & 25 & 30 (8-59) & 37 & 4.918 & 916 \\
\hline & $M$ & 59 & $26(6-60)$ & 37 & 6.668 & 731 \\
\hline \multirow[t]{2}{*}{ Yacuiba } & $\mathrm{F}$ & 35 & $16(5-52)$ & 37 & 6.156 & 838 \\
\hline & $M$ & 24 & $23(7-48)$ & 37 & 3.841 & 781 \\
\hline
\end{tabular}

* D0: día 0 del seguimiento de los pacientes

** CQ-DCQ: clororoquina y desetilcloroquina

*** D2: día 2 del seguimiento de los pacientes 
En el día 21 de seguimiento, un paciente presentó formas asexuales del parásito y siete lo hicieron en el día 28. Se presentó un abandono el día 28 del seguimiento.

La concentración media en sangre de cloroquina y desetilcloroquina en el día 2 antes de la última dosis de cloroquina, fue de $817 \mathrm{ng} / \mathrm{ml}$ (rango: 346,68 - 2.631,15).

Con base en el análisis de supervivencia de KaplanMeier, la incidencia acumulada de falla terapéutica fue de 0,1 (10\%) (IC $\left.{ }_{95 \%}: 0,051-0,19\right)$.

La concentración de cloroquina y desetil-cloroquina en sangre de los ocho pacientes de Guayaramerín en los que se evidenció falla terapéutica, mostró valores inferiores a $70 \mathrm{ng} / \mathrm{ml}$ (cuadro 2).

\section{Discusión}

La resistencia de $P$. vivax a la cloroquina representa un serio problema de salud pública (34); es así que la OMS recomienda cambiar el esquema de tratamiento con cloroquina cuando existe una tasa de falla terapéutica mayor de $10 \%$ evidenciada en el día 28 de seguimiento (35). Sin duda, para muchos programas de malaria, la cloroquina representa uno de los medicamentos de primera línea contra la malaria por $P$. vivax, por lo cual, tanto la vigilancia de la eficacia terapéutica del medicamento como la de la resistencia del parásito al fármaco, son estrategias importantes en el ámbito del control de la enfermedad en muchos países.

La importancia de la determinación de la resistencia a la cloroquina en Bolivia radica en que el $90 \%$ de los casos de malaria son atribuidos a $P$. vivax, teniendo en cuenta, además, que la enfermedad tiene dos zonas de transmisión bien diferenciadas, una al norte del país, en la región amazónica, donde circulan $P$. falciparum y $P$. vivax, y otra en el Sur de Bolivia, donde la malaria se atribuye exclusivamente a $P$. vivax.

El promedio de las concentraciones de cloroquina y desetilcloroquina en sangre, obtenidas en el día 2 de tratamiento antes la última dosis, fue de 815 $\mathrm{ng} / \mathrm{ml}$ en Yacuiba, de $783 \mathrm{ng} / \mathrm{ml}$ en Riberalta y de $817 \mathrm{ng} / \mathrm{ml}$ en Guayaramerín, datos similares a los encontrados en otros estudios, cuyas medias fueron superiores a $700 \mathrm{ng} / \mathrm{ml}$ en el día 2 de seguimiento $(10,36,37)$. Tales resultados muestran una buena absorción gastrointestinal del medicamento en todos los pacientes del estudio, lo que demuestra que no existió interferencia en la absorción del medicamento.

En las localidades de Riberalta y Guayaramerín, se registraron casos de falla terapéutica con frecuencias de $6,2 \%$ y $10 \%$, respectivamente. Los valores registrados de cloroquina y desetilcloroquina en sangre, en el día de la falla terapéutica en todos los pacientes de las dos localidades, fueron inferiores a $70 \mathrm{ng} / \mathrm{ml}$. Sabiendo que la concentración efectiva mínima del medicamento está presente todavía a los 35 días después de su administración (29) y comprobada la buena absorción gastrointestinal del medicamento, tanto en Riberalta como en Guayaramerín, estos resultados muestran que las recaídas no se debieron a resistencia al medicamento, sino a que la concentración del fármaco

Cuadro 2. Medición de cloroquina y de desetilcloroquina en sangre total, en pacientes con falla terapéutica. Guayaramerín y Riberalta

\begin{tabular}{lcccccc}
\hline Localidad & Código & Edad & Peso $\mathbf{( k g )}$ & $\begin{array}{c}\text { Día falla } \\
\text { terapéutica }\end{array}$ & $\begin{array}{c}\text { CQ-DCQ } \\
\text { (ng/ml) D2* }\end{array}$ & $\begin{array}{c}\text { CQ-DCQ } \\
\text { (ng/ml) FT** }\end{array}$ \\
\hline Guayaramerín & G-049 & 13 & 50 & 28 & 980,99 & 44,21 \\
Guayaramerín & G-052 & 21 & 56 & 28 & 644,67 & 65,51 \\
Guayaramerín & G-058 & 22 & 47 & 28 & $1.569,39$ & 49,76 \\
Guayaramerín & G-062 & 25 & 71 & 28 & $1.175,82$ & 47,83 \\
Guayaramerín & G-067 & 18 & 48 & 28 & $1.403,45$ & 43,35 \\
Guayaramerín & G-070 & 36 & 59 & 28 & $1.274,24$ & 69,37 \\
Guayaramerín & G-071 & 32 & 91 & 21 & $2.631,16$ & 44,03 \\
Guayaramerín & G-072 & 25 & 48 & 28 & 574,37 & 65,2 \\
Riberalta & Rib-68 & 9 & 28,7 & 21 & $1.991,19$ & 62,34 \\
Riberalta & Rib-77 & 33 & 68 & 28 & $1.421,06$ & 24,02 \\
Riberalta & Rib-78 & 18 & 64,8 & 28 & $1.429,87$ & 44,29 \\
Riberalta & Rib-82 & 29 & 49,4 & 28 & $1.233,69$ & 49,07 \\
Riberalta & Rib-84 & 21 & 66 & 28 & $1.261,12$ & 54,87 \\
\hline
\end{tabular}

* Cloroquina y desetilcloroquina en el día 2 del seguimiento

** Cloroquina y desetilcloroquina en el día de la falla terapéutica 
estuvo por debajo de la concentración efectiva mínima.

Los valores por debajo de la concentración efectiva mínima antes del día 35 pueden explicarse por diversos factores que interactúan con el citocromo P-450 (CYP-450) (38), como las interacciones medicamentosas, los hábitos dietéticos y las variaciones genéticas del huésped (39-41), las que pueden potencializar el complejo enzimático del citocromo P-450, acelerando el metabolismo de la cloroquina, con su consecuente eliminación prematura.

Sin embargo, para afirmar esto es necesario desarrollar nuevos estudios donde se mida la concentración de la cloroquina y desetilcloroquina en sangre al final de la última dosis del medicamento y al final del seguimiento, en todos los pacientes de un estudio.

En la localidad de Yacuiba, no se reportaron casos de fracaso terapéutico, demostrándose que el fármaco tiene una eficacia in vivo de $100 \%$ para el tratamiento de la malaria por $P$. vivax, dato que confirma el obtenido en la evaluación de Martínez, et al., en 2003, en la misma región (18).

En el contexto suramericano, desde Phillips, et al. (11) en Guyana, donde dos de los tres pacientes presentaron falla terapéutica, se han realizado varios estudios, reportándose desde completa eficacia del medicamento, en los estudios de Castillo, et al., Villalobos-Salcedo, et al., Blair, et al. y Martínez, et al., que evaluaron 44, 73, 33 y 60 pacientes, respectivamente, en diferentes puntos de Suramérica $(17,18,42,43)$, ninguno de los cuales presentó parasitema recurrente en un plazo de 28 días, hasta resistencia comprobada, pero con un riesgo menor de $5 \%$ encontrado en el Perú (15). En un último estudio en Manaus, Brasil, se registró una resistencia del $10 \%$ en 11 de las 109 personas evaluadas, aparentemente con una concentración sérica de $100 \mathrm{ng} / \mathrm{ml}$ de cloroquina, en el día de la falla terapéutica mayor (16).

A pesar de estos hallazgos, no se ha encontrado prueba suficiente como para cambiar el esquema de tratamiento de paludismo por $P$. vivax en la región de las Américas, en donde, como en Bolivia, con estos resultados, la cloroquina junto con la primaquina continúan siendo el esquema de primera línea para tratamiento de $P$. vivax.

\section{Agradecimientos}

El equipo de investigación agradece el apoyo de los equipos regionales del Programa Nacional de
Control de Malaria y de los equipos de trabajo de cada regional; así también, a los trabajadores del CONCAMYT, por su apoyo incondicional en el trabajo. Un agradecimiento especial, a manera de homenaje póstumo, a Ruth Figueroa, quien formó parte del equipo de investigadores.

\section{Conflictos de intereses}

Los autores declaran que no existe conflicto de interés.

\section{Financiación}

Este estudio fue realizado gracias al financiamiento de la United States Agency for International Development (USAID), en el marco de la Iniciativa Amazónica contra la Malaria (IAM) y la Red Amazónica de la Vigilancia de la Resistencia de las Drogas Antimaláricas (RAVREDA), y del Programa Nacional de Malaria del Ministerio de Salud y Deportes de Bolivia.

\section{Referencias}

1. World Health Organization. World Malaria Report 2009. Washington: WHO; 2009. Fecha de consulta: 15 octubre de 2010. Disponible en: http://www.who.int/malaria/world_ malaria_report_2009/en/index.html.

2. Organización Panamericana de la Salud, Organización Mundial de la Salud.142a Sesión del Comité Ejecutivo Malaria: Informe sobre la situación actual CE142/16. Washington, D.C.: OPS/OMS; 2008.

3. Heggenhougen $\mathbf{K H}$, Hackenthal V, Vivek P. The behavioural and social aspects of malaria and its control: An introduction and annotated bibliography. UNDP, World Bank, WHO - Special Programme for Research and Training in Tropical Diseases. Geneva: WHO; 2003.

4. Uhlemann AC, Yuthavong Y, Fidock D. Mechanism of antimalarial drug action and resistance. En: Sherman IW, editor. Molecular approaches to malaria. Washington DC: ASM Press; 2005. p-229-61.

5. Rieckmann KH, Davis DR, Hutton DC. Plasmodium vivax resistance to chloroquine? Lancet. 1989; 2:1183-4. http:// dx.doi.org/10.1128/AAC.48.11.4075-4083.2004

6. Baird JK, Sustriayu Nalim MF, Basri H, Masbar S, Leksana B, Tjitra E, et al. Survey of resistance to chloroquine by Plasmodium vivax in Indonesia. Trans $\mathrm{R}$ Soc Trop Med Hyg. 1996;90:409-11. http://dx.doi.org/10.1016/ S0035-9203(96)90526-X,

7. Baird JK, Basry H, Subianto B, Fryaudff DJ, Mc Elroy PD, Leksana B, et al. Treatment of chloroquine-resistant Plasmodium vivax with chloroquine and primaquine or halofantrine. J Infect Dis. 1995;171:1678-82. http://dx.doi. org/10.1093/infdis/171.6.1678

8. Baird JK, Leksana B, Masbar S, Sutanihardja MA, Fryauff DJ, Subianto B. Whole blood chloroquine concentrations with Plasmodium vivax infection in Irian Jaya, Indonesia. Am J Trop Med Hyg. 1997;56:618-20. 
9. Tulu AN, Webbeg RH, Schellenberg JA, Bradley DJ. Failure of chloroquine treatment for malaria in the highlands of Ethiopia. Trans R Soc Trop Med Hyg. 1996;90:556-7.

10. Teka H, Petros B, Yamuah L, Tesfaye G, Elhassan I, Muchohi S, et al. Chloroquine-resistant Plasmodium vivax malaria in Debre Zeit, Ethiopia. Malar J. 2008;7:220. http:// dx.doi.org/10.1186/1475-2875-7-220

11. Phillips EJ, Keystone JS, Kain KC. Failure of combined chloroquine and high dose primaquine therapy for Plasmodium vivax malaria acquired in Guyana, South America. Clin Infect Dis. 1996;23:1171-3. http://dx.doi. org/10.1093/clinids/23.5.1171

12. Alecrim MC, Alecrim W, Macedo V. Plasmodium vivax resistance to chloroquine (R2) and mefloquine (R3) in Brazilian Amazon Region. Rev Soc Bras Med Trop. 1999;32:67-8. http://dx.doi.org/10.1590/S0037-86821999000100013

13. Soto J, Toledo J, Gutiérrez P, Luzz M, Llinás N, Cedeno $\mathbf{N}$, et al. Plasmodium vivax clinically resistant to chloroquine in Colombia. Am J Trop Med Hyg. 2001;65:90-3.

14. Baird KJ. Resistance to therapies for infection by Plasmodium vivax. Clin Microbiol Rev. 2009;3:508-34. http://dx.doi.org/10.1128/CMR.00008-09

15. Ruebush TK, Zegarra J, Cairo J, Andersen EM, Green M, Pillai DR, et al. Chloroquine-resistant Plasmodium vivax malaria in Peru. Am J Trop Med Hyg. 2003;69:548-52.

16. De Santana Filho FS, Lima-Arcanjo AR, Chehuan YM, Costa MR, Martinez-Espinosa FE, Vieira JL, et al. Chloroquine-resistant Plasmodium vivax, Brazilian Amazon. Emerg Infect Dis. 2007;13:1125-9. http://dx.doi.org/10.3201/ eid1307.061386

17. Castillo CM, Osorio LE, Palma GI. Assessment of therapeutic response of Plasmodium vivax and Plasmodium falciparum to chloroquine in a Malaria transmission free area in Colombia. Mem Inst Oswaldo Cruz. 2002;97:55962. http://dx.doi.org/10.1590/S0074-02762002000400020

18. Martínez E, Yucra WO, Castro V, Figueroa RV, De la Cruz L, Téllez C, et al. Evaluación de la eficacia de la cloroquina para el tratamiento de la malaria por Plasmodium vivax en Yacuiba, Tarija, Bolivia. Cuad Hosp Clin. 2009;54:27-33.

19. Ministerio de Salud y Deportes Bolivia. Dirección General de Servicios de Salud. Unidad de Epidemiología. Tratamiento de la malaria actual. La Paz: Ministerio de Salud y Deportes Bolivia; 2007. p. 1-4.

20. World Health Organization. Reviewing and changing the antimalarial treatment policy. En: Malaria case management: Operations manual. Ginebra: WHO; 2009. p. 66. Fecha de consulta: 30 de noviembre de 2010. Disponible en: http:// www.who.int/malaria/publications/atoz/malaria_case_ management_operations_manual.pdf.

21. Ministerio de Salud y Deportes, Programa Nacional de Malaria. Situación Actual de la malaria en Bolivia. Parte Epidemiológico. La Paz: Ministerio de Salud y Deportes; 2009.

22. Garron A, Mollinedo R. La malaria en Bolivia. Revista Médica La Paz. 2000;7:57-61.

23. Organización Panamericana de la Salud, Organización Mundial de la Salud. Protocolo genérico para estudios in vivo de eficacia de los medicamentos antimaláricos en las Américas. Washington: OPS/OMS, 2003. Fecha de consulta: 5 de diciembre de 2009. Disponible en: http:// www.paho.org/spanish/AD/DPC/CD/mal-antimalarials. $\mathrm{htm}$.

24. Organización Panamericana de la Salud, Organización Mundial de la Salud. Guía práctica para estudios in vivo de eficacia de los medicamentos antimaláricos en las Américas. Washington: OPS/OMS; 2003. Serie OPS/DPC/CD/240/03. Fecha de consulta: 18 de junio de 2009. Disponible en: http://www.paho.org/spanish/AD/DPC/CD/guia-practica. htm.

25. Ruebush TK, Marquiño W, Zegarra J, Neyra D, Villaroel $\mathbf{R}$, Ávila JC, et al. Practical aspects of in vivo antimalarial drug efficacy testing in the Americas. Am J Trop Med Hyg. 2003;68:391-7.

26. Jiménez M. Guía práctica del diagnóstico de la malaria. Publicación 154, Serie Documentos Técnicos y Normativos. La Paz: Ministerio de Salud y Deportes; 2010.

27. Bell DJ, Nyirongo SK, Molyneux ME, Winstanley PA, Ward SA. Practical HPLC methods for the quantitative determination of commonantimalarials inAfrica.JChromatogr B Analyt Technol Biomed Life Sci. 2007;847:231-6. http:// dx.doi.org/10.1016/j.jchromb.2006.10.020

28. Baird JK. Effectiveness of antimalarial drugs. N Engl J Med. 2005;352:1565-77. http://dx.doi.org/10.1056/NEJMra 043207

29. World Health Organization. Plasmodium vivax. Methods for surveillance of antimalarial drugs efficacy. Geneva: WHO; 2004. p. 14.

30. Baird JK, Leksana B, Masbar S, Fryauff D, Sutanihardja A, Suradi F, et al. Diagnosis of resistance to chloroquine by Plasmodium vivax: Timing of recurrence and whole blood chloroquine levels. Am J Trop Med Hyg. 1997;56:621-6.

31. Baird JK. Chloroquine resistence in Plasmodium vivax. Antimicrob Agents Chemother. 2004;48:4075-83. . http:// dx.doi.org/10.1128/AAC.48.11.4075-4083.2004

32. Murphy GS, Basri H, Purnomo MS, Andersen EM, Bangs MJ, Mount DL, et al. Vivax malaria resistant to treatment and prophylaxis with chloroquine. Lancet. 1993;341:96-100. http://dx.doi.org/10.1016/0140-6736(93)92568-E

33. Organización Mundial de la Salud. Instrumento y ejercicio para estudios in vivo de eficacia de los medicamentos antimaláricos en las Américas. Ginebra: OMS; 2004. Fecha de consulta: 16 de agosto de 2009. Disponible en: http://www.paho.org/english/ad/dpc/cd/mal-instrument28daylow-2.xls.

34. Baird JK. Neglect of Plasmodium vivax malaria. Trends Parasitol. 2007;23:533-9. http://dx.doi.org/10.1016/j. pt.2007.08.011

35. World Health Organization. Methods for surveillance of antimalarial drug efficacy. Geneva: WHO; 2009. p. 9.

36. Ketema T, Bacha K, Birhanu T, Petros B. Chloroquineresistant Plasmodium vivax malaria in Serbo town, Jimma zone, south-west Ethiopia. Malar J. 2009;30;8:177. . http:// dx.doi.org/10.1186/1475-2875-8-177

37. Sutanto I, Suprijanto S, Nurhayati, Manoempil P, Baird JK. Resistance to chloroquine by Plasmodium vivax at Alor 
in the Lesser Sundas Archipelago in eastern Indonesia. Am J Trop Med Hyg. 2009;81:338-42.

38. Giao PT, de Vries PJ. Pharmacokinetic interactions of antimalarial agents. Clin Pharmacokinet. 2001;40:343-73.

39. Guzmán V, Carmona-Fonseca J. El citocromo P-450 y la respuesta terapéutica a los antimaláricos. Rev Panam Salud Pública. 2006;19:9-22. http://dx.doi.org/10.1590/ S1020-49892006000100003

40. Blair S, Carmona J, Correa A. Malaria en niños: relación entre nutrición e inmunidad. Rev Panam Salud Pública. 2002;11:5-14. http://dx.doi.org/10.1590/S102049892002000100002
41. Guengerich FP. Influence of nutrients and other dietary materials on cytochrome P-450 enzymes. Am J Clin Nutr. 1995;61(Suppl.3):651S-8S

42. Villalobos-Salcedo JM, Tada MS, Kimura E, Menezes J, Pereira-da-Silva LH. In-vivo sensitivity of Plasmodium vivax isolates from Rondonia (western Amazon region, Brazil) to regimens including chloroquine and primaquine. Ann Trop Med Parasitol. 2000;94:749-58.

43. Blair ST, Tobón AC, Echeverri MR, Álvarez GS, Carmona JF. Adecuada respuesta clínica y parasitológica de Plasmodium vivax a la cloroquina en Colombia (Turbo, Antioquia), 2001. Infectio. 2002;6:21-6. 